\title{
IMMEDIATE IMPLANT PLACEMENT WITH IMMEDIATE PROVISIONALIZATION INTO EXTRACTION SOCKETS WITH LABIAL PLATE DEHISCENCE DEFECTS WITHIN THE MAXILLARY ESTHETIC ZONE. A CLINICAL CASE SERIES STUDY
}

\author{
Merna Yehia Ghoneim*, Niveen Askar ${ }^{* *}$, Hisham Fattouh ${ }^{* * *}$ and Mohamed Atef ${ }^{* * *}$
}

\begin{abstract}
Aim: The aim of this study was to demonstrate the effect of immediate implant placement with immediate loading combined with GBR using a mix of autogenous bone and xenograft with a collagen membrane in type 2 extraction sockets on amount and level of labial Plate of bone reconstruction, primary stability and pink esthetic score (PES) after 6 months post-operative
\end{abstract}

Methodology: A total of 15 patients with type 2 extraction sockets, underwent a traumatic extraction of their teeth in maxillary esthetic zone from right second premolar to left second premolar, and received immediate implant placement with guided bone regeneration that achieved by harvesting autogenous bone from chin and mixed with xenograft and placement of a collagen membrane inside the socket without flap elevation, the graft and membrane were fixed by a provisional restoration that screw-retained on the implant. Outcome measures were: amount of graft remodeling primary stability and PES 6 months post-operative.

Results: Mean bone level immediately post-operative was $3.33+0.68$ that decreased to $2.87+0.62 \mathrm{~mm} 6$ months later the difference was statistically significant $(\mathrm{p}<0.001)$, mean PES immediately postoperative was $9.85+1.07$ that increased to $12.85+0.80$ six months postoperative the difference was statistically significant $(\mathrm{p}<0.001)$, mean stability immediately postoperative was $65.6+3.2$ that increased to $73.7+2.4$ six months postoperative the difference was statistically significant $(\mathrm{p}<0.001)$

Conclusion: Guided bone regeneration with immediate implantation and loading in type 2 sockets has a significant influence on labial plate reconstruction, PES enhancement and primary stability elevation.

\footnotetext{
* Master Implantology, Faculty of Dentistry, Cairo University

** Professor, Oral and Maxillofacial Surgery Department, Faculty of Dentistry, Cairo University

*** Assistant Professor, Oral and Maxillofacial Surgery Department, Faculty of Dentistry, Cairo University
} 


\section{INTRODUCTION}

Oral health and oral health care are very important to maintain proper mastication, digestion, phonation, appearance, and psychological wellbeing. The loss of one or more teeth due to any reason may adversely affect the oral health with an affected appearance being the most serious consequence for the patient a nd prime reason cited by the patient for seeking prosthodontics treatment. Tooth loss can occur for a variety of reasons which include congenital absence, trauma, diseases of the dentition (e.g., caries or periodontal disease), as well as mechanical failure. Tooth loss can also occur secondary or concomitantly to various systemic diseases such as cancer, cardiovascular disease, diabetes mellitus, and osteoporosis. Therefore, it is important to not only maintain good oral hygiene, but also overall health. Jemin Kim and Salomon Amar (2006) (1)

There are three basic approaches to replace a missing tooth or teeth including removable dental prosthesis, fixed dental prosthesis, and dental implants. Each alternative has its own benefits and shortcomings. It is important to consider the patient's financial, medical, and emotional condition for the best treatment. James Hupp et al. $2017^{(2)}$

Most advanced way to replace missing teeth is dental implant which is designed to replicate the natural tooth root and crown of the natural tooth. This procedure preserves the gingival mucosa and bone with no damage to adjacent teeth. Conventional procedure for implant placement involves extraction of offending tooth, waiting 2-4 months for extraction socket to heal, insertion of implant, and again waiting for 3-6 months for integration of implant with surrounding bone; after this procedure, another surgery is necessary to expose the implant and to place a prosthetic abutment Taking into consideration the prosthetic treatment, the patient had to wait up to 8-12 months for a lost tooth to be replace [James Hupp et al.
2017] ${ }^{(2)}$. Because of these shortcomings related to conventional technique, strategies were developed to substantially shorten the entire treatment by placement of implant immediately after extraction of tooth followed by immediate loading of implant with prosthesis [Ashman 2000] ${ }^{(3)}$

The loss of tooth in the esthetic area is often a traumatic experience for the patient. Patients may suffer real or perceived detrimental effects following the loss of one or more teeth. Dental implants offer the most effective solutions to replace the missing teeth and achieve the ultimate satisfaction to the patient. Recently, immediate implant placement after extraction of tooth with immediate loading has become more common. The advantages of this procedure include fewer surgical interventions, reduction in overall treatment time, reduced soft and hard tissue loss, and psychological satisfaction to the patient. Immediately placed and immediate loading implants are more predictable and successful than before however, this approach cannot be applied to every immediate implant patient. In comparison to conventional implant treatment, the immediate loading procedure requires more chair-side time at the time of implant placement for both the restorative dentist and the patient. Careful patient screening and selection are required when an immediate implant placement with immediate loading procedure is a treatment consideration. [Mayank Singh et al. 2015] ${ }^{(4)}$

\section{PATIENTS AND METHODS}

This study was conducted in a hospital based post-graduate oral implantology program (Cairo University- Kasr El Einy) and consisted of 15 patients from Kasr El Eini outclinics.

\section{Inclusion criteria}

- Patients indicated for immediate implant placement with presence of partial or complete loss (type 2 sockets) of labial bone plate and 
intact soft tissue based on Elian classification of extraction sockets (Elian etal 2007) ${ }^{(5)}$

- Patients with good systemic health and good oral hygiene

- Maxillary esthetic zone from (second bicuspid to second bicuspid)

- Endodontic lesions that affected the integrity of the facial plate (dehiscence or absence)

\section{Exclusion criteria}

- General medical or psychiatric contraindications

- Pregnancy

- Patients with local or generalized healing limitations

- Uncontrolled Diabetes mellitus

- Smoking

- Vitamin D deficiency

- Type 3 extraction sockets (bone and soft tissue defects)

- Bruxism or other Para functional habits

- Compromised soft tissue conditions.

They were treated with immediate implant placement with bone graft (Xenograft,Geistlich Bioss, Germany) collagen membrane(Biomet, Zimmer, France) and loading with temporary crown. All patients signed a consent form and asked for lab. Investigations of hba1c and cbc. Impression was taken with a hydrocolloid material (silaxil, LASCOD, Italy) and mounted with a maxillary face bow. Patients were diagnosed radiographically using cbct to determine the amount of buccal bone loss (type II socket) whether the loss is limited to the coronal part of the labial bone or extended to the middle or apical part of it. The inclusion criteria were : extraction type 2 sockets with intact palatal bone and healthy gingival tissue with no recession or periodontal diseases, patients with good systemic health. The teeth included are from maxillary (right second premolar to left second premolar) partial or complete loss of buccal plate of bone. The execlusion criteria were general medical contraindications, uncooperative patients, destructive parafunctional habits, bruxism, diabetes and type III sockets. The surgical protocol necessitates atraumatic extraction without flap elevation and then the amount of bony deficiency in the labial bone was examined using periodontal probe. Proper socket debridement was performed prior to implant placement. Osteotomy was drilled for immediate implant placement with palatal bias to avoid injury of labial plate of bone and to provide a room for bone and membrane placement to allow guided bone regeneration to occur. The implant's clean dentis, Korea) engaged the apical palatal bone or/ and the side walls in larger sockets like premolars to gain an adequate stability. The implant shoulder was placed at from 3 to $4 \mathrm{~mm}$ maximum to the free gingival margin. $35 \mathrm{~N}$ initial stability was gained by engagement the apical palatal part of the lateral incisor to facilitate temporarization. The temporary abutment was roughened outside the patient mouth with fissure bur then screwed on the implant to adjust its proper seating. then the gingival sulcus around the implant was lined by Teflon all around to prevent escape of any temporary material. The hole in the celluloid crown used in temporization was made while the crown being checked on the abutment to locate the site of abutment and crown pick up. After adjustment of celluloid crown on the abutment it was removed outside the patient mouth. Temporary crown material (charm temp. Dentkist, Korea) loaded in a gun or flowable composite was injected in celluloid crown, and then placed on the abutment and waiting for setting. After setting of temporary material the abutment and crown was removed as one unit. The celluloid crown was removed and finishing of the crown was done to eliminate any rough surface especially at the site of gingival contour. Addition of flowable composite might be needed to fill any 
gap between abutment and fabricated crown and to adjust the anatomy of it. Rubber cups eventually used for polishing in combination with buff wheel and goat hair stones. This temporary crown was fabricated having the subgingival contour that conformed to the pre-extration state of tooth root cervix so that it could support the soft tissue emergence profile, protect blood clot and contain bone graft and membrane.

Autogenous bone graft was harvested from the chin to be mixed with xenograft (biooss) and saline. The holes in donor site were filled with gel foam and sutured by prolyne 4-0 .an absorbable collagen membrane (BioMend Zimmer) was trimmed and contoured to conform the labial bone deficiency that previously assessed. The membrane was placed against the internal surface of residual labial bone and the gap between the membrane and the implant was filled with the bone particles. After achieving this, fabricated temporary crown was reinserted using hand torque to ensure adequate support of the soft tissue and gingival contour. The patient was given post-operative antibiotic therapy and seen about one week after surgery to inspect the healing during follow-up period. A healing period of 4 month was specified for the patient to allow buccal plate of bone reconstruction

\section{RESULTS}

At the coronal side: Mean bone level immediately post-operative was $3.69 \pm 0.51$ that decreased to $2.64 \pm 0.51 \mathrm{~mm}$ six months postoperative. Mean reduction from immediate to 6 months after was $0.63 \mathrm{~mm}$ representing $17.1 \%$ from the baseline. This difference was statistically significant $(\mathrm{p}<0.001)$

At the middle side: Mean bone level immediately post-operative was $2.90 \pm 0.63$ that decreased to $2.64 \pm 0.51 \mathrm{~mm}$ six months postoperative. Mean reduction from immediate to 6 months after was $0.27 \mathrm{~mm}$ representing $9.3 \%$ from the baseline. This difference was statistically significant $(\mathrm{p}=0.002)$

At the apical side Mean bone level immediately post-operative was $3.38 \pm 1.11$ that decreased to 2.93 $\pm 1.0 \mathrm{~mm}$ six months postoperative. Mean reduction from immediate to 6 months after was $0.45 \mathrm{~mm}$ representing $13.4 \%$ from the baseline. This difference was statistically significant $(\mathrm{p}<0.001)$ (Figure 1a\&b)

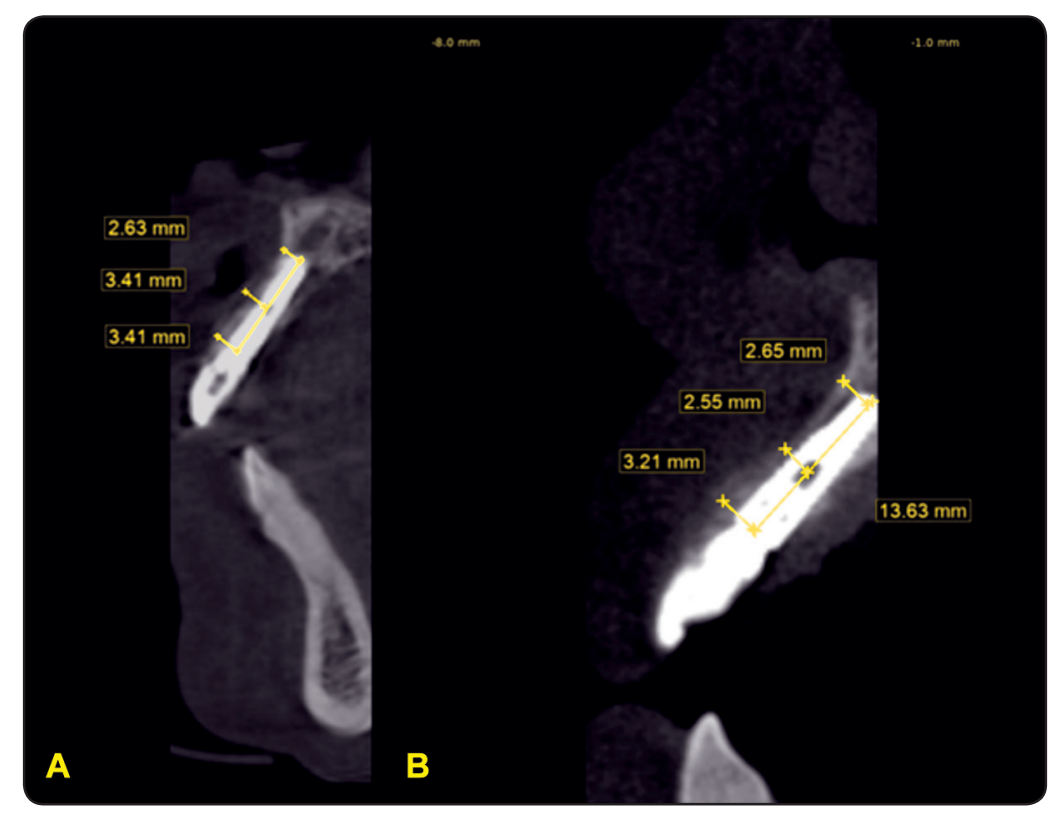

Fig. (1) (a) : immediate postoperative cbct

(b) : cbct after 6 months 


\section{Overall}

Mean bone level immediately post-operative was $3.33 \pm 0.68$ that decreased to $2.87 \pm 0.62$ $\mathrm{mm}$ six months postoperative. Mean reduction from immediate to 6 months after was $0.45 \mathrm{~mm}$ representing $13.6 \%$ from the baseline. This difference was statistically significant $(\mathrm{p}<0.001)$

Mean PES immediately post-operative was $9.85 \pm 1.07$ that increased to $12.85 \pm 0.80$ six months postoperative. Mean increase from immediate to 6 months after was 3 representing 30.5\% from the baseline. This difference was statistically significant $(\mathrm{p}<0.001)$ (figure $2 \mathrm{a} \& \mathrm{~b})$

Mean stability immediately post-operative was $65.6 \pm 3.2$ that increased to $73.7 \pm 2.4$ six months postoperative. Mean increase from immediate to 6 months after was 8.1 representing $12.3 \%$ from the baseline. This difference was statistically significant $(\mathrm{p}<0.001)$ (figure 3)
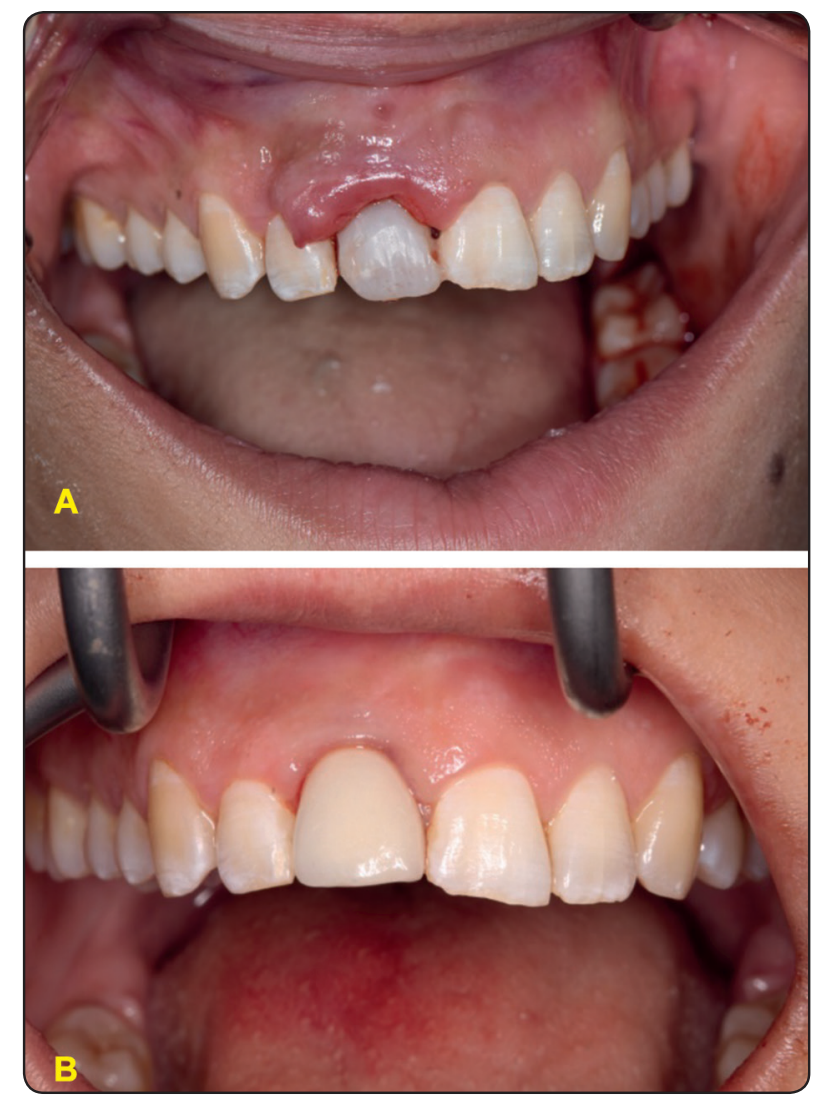

Fig. (2) : (a) Pink esthetic score immediate post-operatively and (b) at 6 months post-operatively

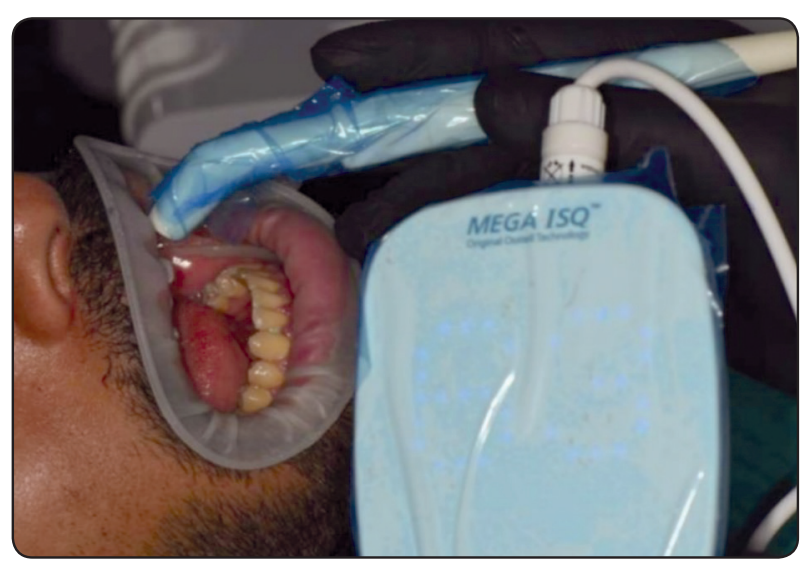

Fig. (3) implant stability quotion

\section{DISCUSSION}

The immediate implant placement technique is currently a routine procedure with success rates like those for the placement of a conventional implant. Immediate placement of an implant in a fresh socket in the aesthetic area is a complex process and involves great dexterity and need a good surgical skills and experience to achieve a good esthetic result. (Monish Bhola, et al. 2015) ${ }^{(8)}$

In the present study the treatment protocol combines the concept of socket preservation technique and immediate implant placement with provisional restoration. This case series study of 15 patients with a type 2 extraction socket demonstrate the immediate implant placement with grafting and absorbable collagen membrane placement with immediate provisionalization results in reformation of labial plate of bone with dehiscence defect at 6 to 9 months. the aim of this study to decrease the number of surgical steps required to prepare the extraction socket with buccal plate of bone deficiencies to receive an implant.

The implant system used in this study (dentis, $S$ clean) tapered design to aid gaining primary stability, biomend absorbable collagen membrane was used and a mix of autogenous and xenograft was placed between the implant and internal surface of the membrane and they fixed and contained 
inside the socket by custom made provisional restoration using celluloid crowns and temporary crown materials or composite resin material.

Atraumatic tooth extraction was done to decrease the amount of labial bone resorption and maintain the gingival contour as reported by Rudys Rodolfo De Jesus Tavarez et al. in $2013^{(6)}$, it is observed that the atraumatic tooth extraction combined with immediate implant placement presents clinical results that provides harmony and aesthetics of the gum line, this is attributed to preservation of the remaining labial plate of bone during extraction that could be easily fractured and maintain the gingiva intact without laceration .

Alves and Neves. $2009^{(7)}$, reported that Because the tapered implant design has its self- tapping property this surgical technique was developed to optimize the bone compaction effect on the coronal third of the implant, resulting in improving bone density and providing better primary stability values (> or $=70$ ISQ, via the Osstell device).

It was observed that the main advantages of tapered or root form implant were that they were ideal for immediate implant placement and welldesigned for narrow gaps with root proximity of adjacent teeth, and they were highly indicated for soft bone for they condense bone locally resulting in increasing primary stability, they also provide less risk of labial plate perforation due to their reduced apical diameter in all directions. implant length should also be highly considered, as the basal or apical and palatal bone of the extraction socket should be engaged properly to gain primary stability without increasing the depth of implant position and that couldn't be achieved unless long implant were used, at least $13 \mathrm{~mm}$ and selecting a diameter of implant that closely matches the extaction socket . Immediate loading could be predictable with the strong bond that is created between the implant surface and the surrounding bone using these implant dimensions and design.
When the patient has a thick soft tissue biotype, fabricating a provisional restoration with immediate implant is the preferred treatment option. This depends on the primary stability which should be $35 \mathrm{~N}$ or higher, If this is not achieved then it is recommended to place the implant with grafting and loaded 3 to 4 months later. The same treatment of socket preservation should be in patients with a thin biotype. To give the clinician the ability to use the extra keratinized tissue that was gained on the occlusal aspect of the socket. During second-stage surgery and at the time of fabricating the provisional crown, it turned out that the $3 \mathrm{D}$ position of the implant is the most effective determinant of implant success in esthetic zone even if the patient had thin gingival biotype. (Monish Bhola, et al. 2015) ${ }^{(8)}$

In this study when the primary stability was less than $35 \mathrm{~N}$ the socket seal provisional abutment was fabricated instead of full anatomy temporary crown , in order to prevent any kind of non-axial loading on the implant. The purpose of socket seal fabrication was to take the advantage of temporarization in creating a proper emergence profile and giving a high pink esthetic score at the time of final prosthesis placement, the 6 months post operative results were the same in either a full anatomy crown or a socket seal regarding the emergence profile and pink esthetic score.

In a study conducted by Nima Naddaf Pour et al. $2018^{(9)} 42$ patients were divided into 22 who underwent immediate implant placement and 20 underwent conventional implant placement to demonstrate the influence of immediate implant placement on pink esthetic score and marginal bone loss compared to delayed one. The mean bone loss was $0.62 \pm 0.44 \mathrm{~mm}$ in the immediate and $0.43 \pm$ $0.39 \mathrm{~mm}$ in the conventional group. The difference was not statistically significant between the two groups, the mean PES was 8.54 (range 6-10) in the immediate and 8.10 (range 6-10) in the conventional group. The difference was not statistically 
significant between the two groups $(P>0.05)$. In the current study a great enhancement of (PES) due to a proper preservation of emergence profile with a highly finished and polished temporary crown was observed at the time of final restoration delivery and a new bone reformation occurred .

Ice cream cone technique is a socket or ridge preservation approach that was applied in type 2 extraction socket in 2014 by Jocelyn H.P. Tan-Chu et al. ${ }^{(10)}$ in retrospective study contained 11 extraction socket. In this technique a collagen membrane was trimmed into ice cream cone shape inserted inside the extraction socket and bone allograft was placed without flap elevation. Two resorbable 4.0-chromic sutures were used to suture and secure the membrane over the site to prevent dislodgement or loss of the particulate bone graft material and blood clot during initial healing. All grafted sites were allowed to heal after 6 months to properly receive dental implants. The reduction in the bucco-lingual dimensional changes were $1.28 \mathrm{~mm}$, while regenerating the lost buccal plate adequately to place an endosseous implant with minor bone grafting. In the present study immediate implant placement with immediate loading accompanied with socket grafting was achieved in type 2 socket, collagen membrane not sutured beacause its fixation and graft containment depends on the placement of temporary crown so there was time reduction of second stage surgery and creating a proper emergence profile at the time of surgery by installing the provisional crown, the amount of graft remodeling was $0.6 \mathrm{~mm}$.

In 2011 a case report study published by ChihLongChen et al. ${ }^{(11)}$ showing the effect of immediate implant placement and provisionalization with simultaneous guided bone regeneration. In this case, a 46 year old woman with high esthetic expectation had a buccal bony dehiscence with a vertical root fracture and inflamed gingiva. The full thickness flap was elevated, bone graft was placed directly on the labial bone defect and in the gap defect inside the extraction socket and the collagen membrane was placed over it and a soft-tissue closure in a non-submerged approach was secured with 5-0 sutures. high implant survival rates and predictable good esthetic outcomes can be achieved with shortterm follow-up. in the current study guided bone regeneration was achieved without flap elevation but may be in the previous study flap elevation was mandatory due to the presence of vertical root fracture to avoid traumatic extraction or soft tissue laceration during tooth removal.

In 2013 a report of 3 cases was done by Waasdrop et al. ${ }^{(12)}$ using a high density Polytetrafluorethylene membrane in guided bone regeneration with immediate implant placement to augment horizontal defects, the advantage of this membrane is that it withstand exposure to the oral cavity and placed with slight approximation without primary closure, the membrane was left in place for 6 weeks and removed easily without anesthesia, the reentry surgery demonstrated complete clinical bone fill with normal healing. The advantage of this technique over the technique in the present study is that PTFE has the property of micro porosity and their pore size makes them impenetrable to bacteria and epithelia cells, however the collagen membrane characteristics in this study are very special because it acts as an effective barrier and give longer retention for 6 to 7 weeks and undergoes complete absorption into surrounding tissue by 8 weeks in addition, it is non-slippery when wet and its rigidity makes is easily applied into the extraction socket to compensate for the missing part of the labial plate of bone, it assists in clot maintenance and stability of the graft materials and does not require a second stage surgery for removal.

Bone grafts used in the present study was mix of autogenous which was harvested from the chin by auto chip maker (ACM) bur mix of 50\% autogenous bone particulated $50 \%$ xenograft is used to fill this gap. Autogenous bone particulated graft was chosen 
for their osteogenic property and biocompatibility and xenografts for its ability for transitional ridge preservation because of their slow resorption rate. In a study done by Maria Zempila et al. $2017^{(13)}$ showed that xenograft and allograft bone materials were used as a bone fillers of gap created after immediate implant placement. And they are divided into 2 goups, after 6 months cbct was done and It was reported that there was a slight reduction in the six month measurement in relation to the buccal bone width, without any significant differences between the two groups while the mean vertical bone reduction in the allograft group (B' experimental group), was almost 4 times larger than that in the xenograft group (A' experimental group) concerning bone fill material, comparing the current study to the study done by Guido Sarnachiaro et al. 2015 ${ }^{(14)}$ where the patients received immediate implant placement in type 2 sockets using allograft as bone fill material, there was no significant difference in graft remodeling whether the graft material was allograft or mix of autogenous and xenograft.

Several factors can influence the amount of labial plate thickness reconstruction such as ,the amount of labial plate loss, preoperative soft tissue inflammation, and periodontal phenotype(tarnow et al 2015) ${ }^{(15)}$. There are anatomical structure to be examined in a single tooth extraction site in the esthetic zone, these anatomical structure are : thickness, height and integrity of the facial bone wall, the height and thickness of palatal bone wall ,the crest width mesially and distally to the extraction site which is measured $3 \mathrm{~mm}$ apical to the cementoenamel junction of the adjacent teeth, the height and inclination of the alveolar ridge, the location of nasopalatine canal, the amount of bone apically and palatally of the root and the mesiodistal size of the resulting single tooth gap post extraction and the height of the alveolar bone of the adjacent teeth. (Daniel Buser et al. 2016) ${ }^{(16)}$ in a study conducted by Hani el Nahass and Suzy N. Naiem $2014{ }^{(17)}$ it was reported that labial bone thickness is thin with a mean thickness between 0.57 and $0.84 \mathrm{~mm}$. accordingly in the current study if the level of bone loss not extended to the middle or apical part of the socket, new bone regeneration was more predictable, the implant position should be (3 to $5 \mathrm{~mm}$ ) from the free gingival margin to achieve a good emergence profile .

The main advantage of the present study was time that saved and flapless surgery that prevented soft tissue reflection and reduced the surgical trauma which in turn accelerated healing time and decreased laceration and scaring resulting from suturing. Moreover, it minimized the surgical steps required if guided bone regeneration was the treatment option, the patient has to undergo first stage surgery that comprises bone and membrane placement then the implant would be placed from 6 to 9 months postoperative with soft tissue graft augmentation that is most probably needed after GBR procedure then the implant would be finally restored .

On the other hand, if there are multiple extraction with type 2 sockets in such cases full thickness flap should be done and socket preservation or guided bone regeneration techniques without simultaneous implant placement are the best treatment option , flapless technique is favorable in type 2 socket when the tooth to be extracted is bounded by adjacent teeth and soft tissue is healthy so, immediate implant placement with guided bone regeneration and immediate temporization would be effective and enhance esthetics, however, in cases required multiple extractions .flap elevation is mandatory for proper curettage and removal of cysts and multiple granulomas and bony reconstruction of fenestrations that are usually existed in such cases.

In 2017 it a RCT study done by Tonetti et al ${ }^{(18)}$. to compare the need for bone augmentation and surgical complication in subjects received immediate implant placement or 12 weeks after extraction (delayed), IMI was unfeasible in $7.5 \%$ of cases. One hundred and 24 subjects were randomized. One implant was lost in the IMI group. IMI required 
bone augmentation in $72 \%$ of cases compared with $43.9 \%$ for delayed while wound failure occurred in $26.1 \%$ and $5.3 \%$ of cases, respectively At 1 year, IMI had deeper probing depths $(4.1 \pm 1.2 \mathrm{~mm}$ versus $3.3 \pm 1.1 \mathrm{~mm}$, greater radiographic bone loss was observed at IMI over the initial 3-year period Inadequate pink aesthetic scores were obtained in $19 \%$ of delayed and in $42 \%$ of IMI implant cases No differences in patient reported outcomes were observed. So, Immediate implant placement should not be recommended when aesthetics are important, IMI should be limited to selected cases. On contrary, based on the results in the present study, it is recommended that whenever the bony dimensions allowed immediate implant with proviosionalization, if it is possible it should be the best line of treatment, since it greatly enhanced the PES and preserve the emergence profile.

In 2014 a case report study was done by Khorshid et $^{\mathrm{a}^{(19)}}$. to document the causes of implant failure after immediate loading, 4 implants were lost during the second week, 5 implants were lost during 3 weeks after loading. It was reported that early failure after immediate loading attributed to poor maxillary bone quality, non-axial loading, high forces delivered from opposing dentition and patient with parafunctional habit. In the current study 2 implants were failed and that could be attributed to parafunctional habits of the patients and that is why careful selection of the patients receiving immediate loading should be highly comsidered, as well as the selection whether to do full anatomical crown or just to preserve the emergence profile with socket seal abutment.

\section{CONCLUSION}

Guided bone regeneration with immediate implantation and loading in type 2 sockets has a significant influence on labial plate reconstruction, PES enhancement and primary stability elevation, provided that the dental implant placed in a correct $3 \mathrm{D}$ position.

\section{REFERENCES}

1. Kim J and Amar S (2006). Periodontal disease and systemic conditions: a bidirectional relationship. Odontology. 94(1): 10-21.

2. Hupp JR 2017. introduction to implant dentistry: a student guide. Journal of Oral and Maxillofacial Surgery. 75(2): $1-100$.

3. Ashman A (2000). Postextraction ridge preservation using a synthetic alloplast. Implant Dentistry. 9(2):168-176.

4. Singh M, Kumar L, Anwar M \& Chand P (2015). Immediate dental implant placement with immediate loading following extraction of natural teeth. National Journal of Maxillofacial Surgery. 6(2):252-

5. Elian N, Cho SC, Froum S, Smith RB, Tarnow DP (2007). A simplified socket classification and repair technique. Practical Procedures of Aesthetic Dentistry. 19(2):99-104.

6. Tavarez RR, Dos Reis WL, Rocha AT, Firoozmand LM, Bandéca MC, Tonetto MR \& Malheiros AS (2013). Atraumatic extraction and immediate implant installation. Journal of International Oral Health. 5(6):113-118

7. Alves CC and Neves M (2009). Tapered implants: from indications to advantages. International Journal of Periodontics \& Restorative Dentistry. 29(2):161-167.

8. Bhola M, Jacobs LC \& Kolhatkar S (2015). Immediate implants for aesthetic success: New guidelines. Journal of the International Clinical Dental Research Organization. 7(3):138-147

9. Pour NN, Ghaedi B \& Sohrabi M (2018). Soft-tissue esthetic outcome of single implants: Immediate placement in fresh extraction sockets versus conventional placement in healed sockets. Journal of Indian Society of Periodontology. 22(3):249-253.

10. Tan-Chu JH, Tuminelli FJ, Kurtz KS, Tarnow DP (2014). Analysis of Buccolingual Dimensional Changes of the Extraction Socket Using the "Ice Cream Cone" Flapless Grafting Technique. the International Journal of Periodontics \& Restorative Dentistry. 34(3):399-403.

11. Chen CL, Chang CL \& Lin SJ (2011). Immediate implant placement and provisionalization with simultaneous guided bone regeneration in the esthetic zone. Journal of Dental Sciences. 6(1):53-60.

12. Waasdorp J, Feldman S (2013). Bone Regeneration Around Immediate Implants Utilizing a Dense Polytetrafluoroeth- 
ylene Membrane Without Primary Closure: A Report of 3 Cases. Journal of Oral Implantology. 39(3):355-361.

13. Zempila M, Doufexi AE, Batas L \& Konstantinidis A (2017). The role of graft material in bone resorption after immediate implant placement. Clinical Oral Implants Research. 28(14):512-512

14. Sarnachiaro GO, Chu SJ, Sarnachiaro E, Gotta SL \& Tarnow DP (2015). Immediate Implant Placement into Extraction Sockets with Labial Plate Dehiscence Defects: A Clinical Case Series. Clinical Implant Dentistry and Related Research. 18(4):821-829.

15. Chu SJ, Sarnachiaro GO, Hochman MN \& Tarnow DP(2015). Subclassification and Clinical Management of Extraction Sockets with Labial

16. Buser D, Chappuis V, Belser UC, Chen S (2016). Implant placement post extraction in esthetic single tooth sites: when immediate, when early, when late?. Periodontology 2000. 73(1): 84-102.

17. El Nahass $\mathrm{H}$ and Naiem SN (2014). Analysis of the dimensions of the labial bone wall in the anterior maxilla: a cone-beam computed tomography study. Clinical Oral Implants Research. 26(4):57-61.

18. Tonetti MS, Cortellini P, Graziani F, Cairo F, Lang NP, Abundo R, Conforti GP, Marquardt S, Rasperini G, Silvestri M, Wallkamm B \& Wetzel A (2017). Immediate versus delayed implant placement after anterior single tooth extraction: the timing randomized control trail. Journal of Clinical Periodontology. 44(2):215-224

19. Khorshid HE, Hamed HA, Aziz EA (2014). Complications, risk factors, and failures of immediate functional loading of implants placed in the completely edentulous maxillae: a report of 3 consecutive cases. Implant Dent. 23(2):125-131. 